\title{
Simulation on Magnetic Field Characteristics of Permanent-Magnet Seed-Metering Device
}

\author{
Jing Wang, Jianping Hu, Qirui Wang, and Xun Wang \\ Key Laboratory of Modern Agricultural Equipment and Technology, \\ Ministry of Education \& Jiangsu Province, Jiangsu University, \\ High-tech Key Laboratory of Agricultural Equipment \& Intelligentization \\ of Jiangsu Province, Zhenjiang 212013, China \\ hujp@ujs.edu.cn
}

\begin{abstract}
The cylindrical permanent magnet is the core part of the permanent-magnet seed-metering device, it can absorb single magnetic powder coated seed. The first, the magnetic induction intensity model of any point in the cylindrical permanent magnet external was established based on the equivalent current model of permanent magnet. The second, the mathematical formula was derived using the Biot-Savart law and the superposition principle of magnetic field. Then its magnetic induction intensity was calculated by multiple numerical integration function of MATLAB, and the characteristics and distribution were obtained. On this basis, the simulation and analysis of the permanent magnet with height $7 \mathrm{~mm}$ and diameter $2 \mathrm{~mm}$ was finished. The results showed that the closer the field point was to the permanent magnet end face, the stronger the magnetic induction intensity was, and the magnetic induction intensity at the edge of end face was stronger than at the center. So the diameter of the cylindrical permanent magnet should be less than magnetic powder coated seed, meanwhile, the air gap between the permanent magnet end face and the magnetic powder coated seed should be smaller as much as possible or the magnetic powder coated seed directly contacted with the permanent magnet end face.
\end{abstract}

Keywords: Magnetic seed-metering device, Permanent magnet, Magnetic field analysis, Numerical integration, Magnetic induction intensity, Equivalent current model of permanent magnet.

\section{Introduction}

In recent years, concerning the problem of accurate sowing for the small seed, the literature [1-3] carried out the research about Magnetic Precision Metering Technology which used electromagnet as the seed-metering component of the metering device. In the literature [4], a permanent-magnet seed-metering device was proposed and the simulation and experiment of sowing performance was studied. The structure diagram of the permanent- magnet seed-metering device was showed in Figure 1. The seed-metering component was a cylindrical permanent magnet. The permanent magnet was installed in the device which can make the permanent magnet to throw out and draw back. This device was installed in the drum. With the drum rotating, the magnetic powder coated seeds were absorbed by seed-metering components when it threw out, and were metered when it drew back. In order to analyze the characteristics and 
distribution of magnetic field about the cylindrical permanent magnet, the paper adopted the equivalent current model of permanent magnet to derive and establish the mathematical model which can be used for calculating the magnetic induction intensity of any point in the permanent magnet external. The mathematical model of magnetic induction intensity about the permanent magnet can be calculated by the multiple numerical integration function of MATLAB, and the distribution can be expressed by the visualization function.

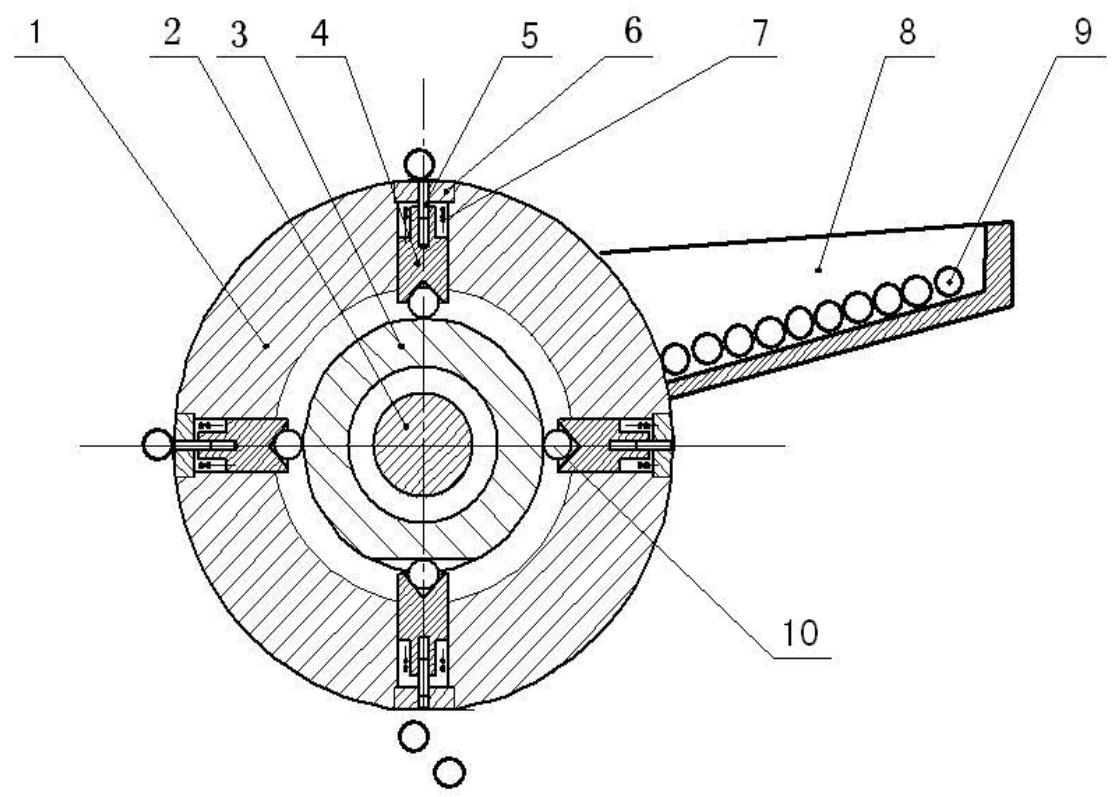

0

0

1. Drum 2. Drive shaft 3. Cam guides 4. Magnet jacket 5. Cylindrical permanent magnet 6. Cover plate 7. Spring 8.Seed box 9.Magnetic powder coated seed 10. Steel ball

Fig. 1. Structural diagram of roller-type metering device

\section{Mathematical Model of the Magnetic Induction Intensity of any Point in the Cylindrical Permanent Magnet External}

\subsection{Analytic Derivation of the Magnetic Induction Intensity of the Cylindrical Permanent Magnet}

At present, the methods of studying the distribution of magnetic field of the cylindrical permanent magnet are magnetic dipole and empirical formula. The magnetic dipole method requires the radius of field point much larger than the cylindrical magnet's, so its error is large when the radius of the cylinder cannot be ignored. The empirical 
formula can only estimate the magnetic induction intensity on the cylinder axis, and the experience factor in the empirical formula can only be got by a large number of experiments, so the method is less accurate. In order to seek an easy and accurate algorithm for magnetic field of the cylindrical permanent magnet, based on the equivalent current model of permanent magnet, the mathematical model was derived to calculate magnetic induction intensity of any point in the cylindrical permanent magnet external. The equivalent current model of permanent magnet can be considered that the external magnetic field of the permanent magnet was generated from the bound surface current of magnet side surface. The current density of bound surface was:

$$
J_{m}=B_{r} / \mu_{0} .
$$

Where: $J_{m}$ - the current density of bound surface; $B_{r}$-remnant magnetization; $\mu_{0}$-vacuum permeability, $\mu_{0}=4 \pi \times 10^{-7} \mathrm{H} / \mathrm{m}$.

When a cylindrical permanent magnet with radius $r_{0}$ and height $h$ was magnetized in one direction and uniformly saturated, the magnetic induction intensity of any point in external space was only excited by the surface of closed current loop of the permanent magnet. Considering the loop current as $I$, the current density of arbitrary plane in parallel with the plane $x o y$ was $J_{m}=I / h$.

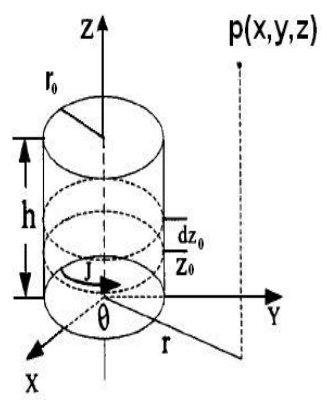

Fig. 2. Parameters of cylinder permanent magnet

The cylindrical coordinate was taken, a point inside the magnet was represented with $\left(r_{0}, \theta, z_{0}\right)$, and the coordinate system was established, as shown in Figure 2. Considering the thin layer consisted of plane $z$ and $z+d z$, the magnetic induction intensity of any point $p(x, y, z)$ in its external space was generated by current loop $J_{m} d z$ to $d \vec{B}$. According to Bio-Savart law and the superposition principle of magnetic field, the magnetic induction intensity of any point $p(x, y, z)$ outside the cylinder was: 


$$
\vec{B}=\frac{\mu_{0}}{4 \pi} \oint_{l} \frac{I d \vec{l} \times\left(\vec{r}-\overrightarrow{r^{\prime}}\right)}{\left|\vec{r}-\overrightarrow{r^{\prime}}\right|^{3}}
$$

Where: $\vec{r}$ - the radius vector of source point $\left(x_{0}, y_{0}, z_{0}\right) ; \vec{r}^{\prime}$ - the radius vector of field point $p(x, y, z)$.

In cylindrical coordinate, a point $M\left(x_{0}, y_{0}, z_{0}\right)$ on the surface of the cylinder can be expressed as $M\left(r_{0}, \theta, z_{0}\right)$, that is, $\left\{\begin{array}{l}x_{0}=r_{0} \cos \theta \\ y_{0}=r_{0} \sin \theta \\ z_{0}=z_{0}\end{array}\right.$.

$$
d \vec{l} \times r_{0}=\left|\begin{array}{ccc}
i & j & k \\
-r_{0} \sin \theta d \theta & r_{0} \cos \theta d \theta & 0 \\
x-r_{0} \cos \theta & y-r_{0} \sin \theta & z-z_{0}
\end{array}\right|
$$

$$
\text { Order: } K=\left[\left(x-r_{0} \cos \theta\right)^{2}+\left(y-r_{0} \sin \theta\right)^{2}+\left(z-z_{0}\right)^{2}\right]^{3 / 2}
$$

Generated from current loop, the magnetic induction intensity $\vec{B}$ in $x, y, z$ directions were:

$$
\left\{\begin{array}{c}
d B_{x}=\frac{\mu_{0} J_{m} d z_{0}}{4 \pi} \int_{0}^{2 \pi} \frac{\left(z-z_{0}\right) r_{0} \cos \theta}{K} d \theta \\
d B_{y}=\frac{\mu_{0} J_{m} d z_{0}}{4 \pi} \int_{0}^{2 \pi} \frac{\left(z-z_{0}\right) r_{0} \sin \theta}{K} d \theta \\
d B_{z}=\frac{\mu_{0} J_{m} d z_{0}}{4 \pi}\left\{\int_{0}^{2 \pi} \frac{-r_{0}\left(x-r_{0} \cos \theta\right) \cos \theta}{K} d \theta\right. \\
-\int_{0}^{2 \pi} \frac{r_{0}\left(y-r_{0} \sin \theta\right) \sin \theta}{K} d \theta
\end{array}\right\}
$$

The space magnetic induction intensity of the cylindrical permanent magnet was: 


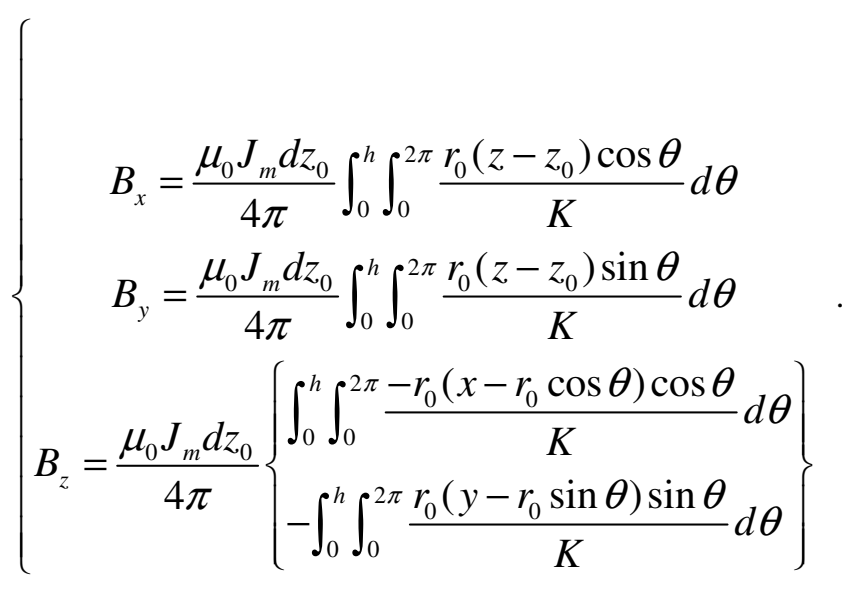

substituted (1) into (6)

$$
\left\{\begin{array}{c}
B_{x}=\frac{B r d z_{0}}{4 \pi} \int_{0}^{h} \int_{0}^{2 \pi} \frac{r_{0}\left(z-z_{0}\right) \cos \theta}{K} d \theta \\
B_{y}=\frac{B r d z_{0}}{4 \pi} \int_{0}^{h} \int_{0}^{2 \pi} \frac{r_{0}\left(z-z_{0}\right) \sin \theta}{K} d \theta \\
B_{z}=\frac{B r d z_{0}}{4 \pi}\left\{\int_{0}^{h} \int_{0}^{2 \pi} \frac{-r_{0}\left(x-r_{0} \cos \theta\right) \cos \theta}{K} d \theta\right. \\
-\int_{0}^{h} \int_{0}^{2 \pi} \frac{r_{0}\left(y-r_{0} \sin \theta\right) \sin \theta}{K} d \theta
\end{array}\right\} .
$$

\subsection{Calculation of the Magnetic Induction Intensity Using MATLAB}

When the integral expression of function was known, it can be solved with Newton Leibniz formula in theory, but it was not practical in the calculation of the magnetic field, for integral functions of most functions can not be found. A lot of command functions of the numerical integration can be provided by MATLAB, and they can be called based on the actual situation, not only convenient but also accurate. The integral command used in this paper was dblquad. Actually, the dblquad was achieved by calling quad repeatedly. The principle of quad was adaptive Simpson quadrature method. If using the quadratic interpolation polynomial--the area of the curved trapezoidal surrounded by the parabola $y=g(x)$ approximate instead of the area of the curved trapezoidal surrounded by $y=f(x)$, the integral formula was called Simpson Formula: 


$$
I=\int_{a}^{b} f(x) d x \approx \frac{b-a}{6}\left[f(a)+4 f\left(\frac{a+b}{2}\right)+f(b)\right]
$$

The interval $[a, b]$ was divided to $K$ equally and $n=2^{k}$ subinterval was obtained, then the composite Simpson formula further improved was:

$$
I=\sum_{i=0}^{n-1} \int_{x_{i}}^{x_{i+1}} f(x) d x \approx s_{n}=\frac{h}{6} \sum_{i=0}^{n-1}\left[f\left(x_{i}\right)+4 f\left(\frac{x_{i}+x_{i+1}}{2}\right)+f\left(x_{i+1}\right)\right] \text {. }
$$

When the variable step Simpson integration method was used to calculate the double integral, the numerical integration approach was to divide the double integral

$$
I=\int_{b}^{a} d x \int_{y_{1}(x)}^{y_{2}(x)} f(x, y) d y
$$

Into

$$
g(x)=\int_{y_{1}(x)}^{y_{2}(x)} f(x, y) d y
$$

And

$$
I=\int_{b}^{a} g(x) d x
$$

In MATLAB, the model in (7) was written into M-file of fun $\left(\theta, z_{0}\right)$, and the integral of the model was realized by $\mathrm{B}=\operatorname{dblquad}(f u n, a, b, c, d$, tol). Where $a, b$ was the upper and lower limit of $\theta ; c, d$ for was the upper and lower limit of $z_{0}$ separately; tol was integration accuracy (default accuracy was 10e-6 ).

\section{Simulation and Analysis the Magnetic Induction Intensity of the Cylindrical Permanent Magnet}

The magnetic coated and pelletized seeds, about $3 \mathrm{~mm}$ in diameter, were used in the permanent-magnet seed-metering device. In order to further simulate, a cylindrical magnet with diameter $2 \mathrm{~mm}$ and length $7 \mathrm{~mm}$ was selected.

Figure 3(a) showed the gradient changes in the $x$ direction of the magnetic induction intensity with the cylindrical permanent magnet in the plane with $y=0,-5 \mathrm{~mm}<x<5 \mathrm{~mm}$, $7 \mathrm{~mm}<z<10 \mathrm{~mm}$; Figure $3(b)$ showed the gradient changes in the $x$ direction of the magnetic induction intensity with the cylindrical permanent magnet in the plane with $z=7 \mathrm{~mm},-5 \mathrm{~mm}<x<5 \mathrm{~mm},-5 \mathrm{~mm}<y<5 \mathrm{~mm}$; Figure $3(c)$ showed the gradient changes in the $z$ direction of the magnetic induction intensity with the cylindrical permanent magnet in the plane with $y=0,-5 \mathrm{~mm}<x<5 \mathrm{~mm}, 7 \mathrm{~mm}<z<10 \mathrm{~mm}$; Figure $3(d)$ showed 
the gradient changes in the $z$ direction of the magnetic induction intensity with the cylindrical permanent magnet in the plane with $z=7 \mathrm{~mm},-5 \mathrm{~mm}<x<5 \mathrm{~mm}$, $-5 \mathrm{~mm}<y<5 \mathrm{~mm}$.

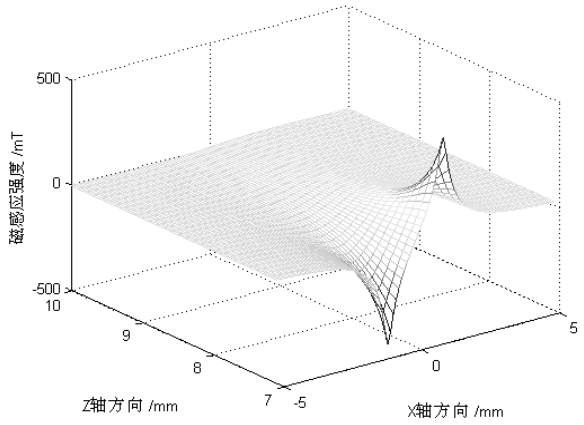

a

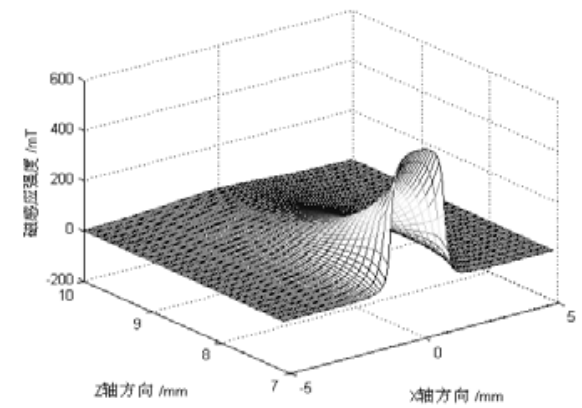

c

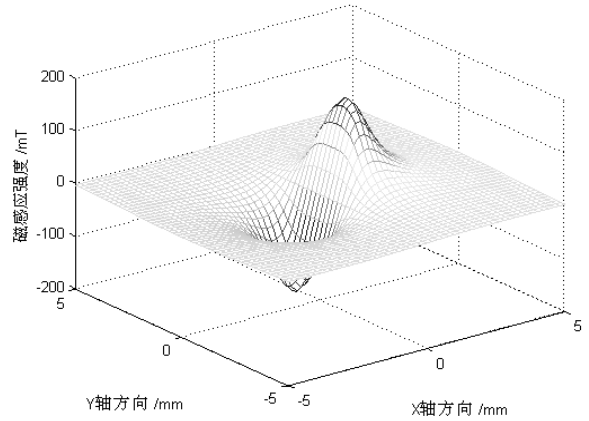

$b$

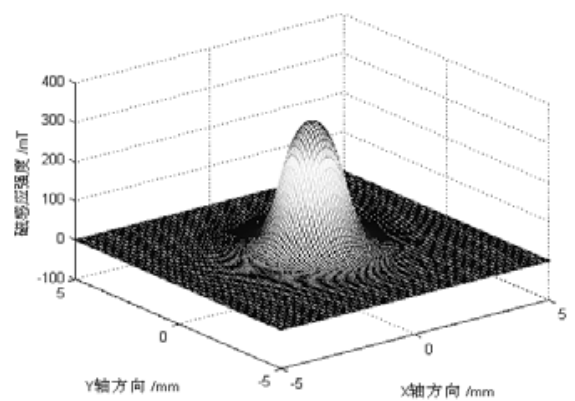

$d$

Fig. 3. Distribution of Magnetic Induction Intensity

The distributions of $B_{x}, B_{z}$ in $x o z$ plane were showed in figure $3(a)$ and figure $3(c)$. The $B_{x}$ reached a maximum value at the $x=1 \mathrm{~mm}$ or $x=-1 \mathrm{~mm}$, zero at the $x=0$, and had a rapidly decay then tended to zero within the radius; $B_{z}$ reached a maximum value on the axis of a cylinder, and the magnetic induction intensity in the diameter range was relatively larger, decaying rapidly outside; the value of $B_{x}$ and $B_{z}$ had rapidly decay in the $Z$-axis direction and tended to zero. The distributions of $B_{x}$ and $B_{z}$ on the end face of the permanent magnet were showed in figure $3(b)$ and figure $3(d)$. The magnetic induction intensity was concentrated within a radius of the cylinder; and it was about zero at the place larger than the radius. 


\section{Conclusions}

1) The paper adopted the equivalent current model of permanent magnet and the Bio-Savart law to derive and establish the mathematical model which can be used for calculating the magnetic induction intensity of any point in the permanent magnet external. The calculated value would not produce errors for the method itself, because the magnetic induction intensity of field point only depended on all source points and the magnetic parameter of permanent magnet was constant. Compared to the magnetic dipole and the empirical formula, this method was more accurate.

2) Using adaptive Simpson quadrature method and the numerical integration function of MATLAB, the precise calculation about the magnetic induction intensity of any point in the permanent magnet external was realized. Meanwhile, the complex derivation process of the discrete integral formula was eliminated and the problems of stability, accuracy and overflow were avoided in the integration formula of recursive process.

3) The simulating calculation results showed that the space magnetic induction intensity of the cylindrical permanent magnet was concentrated on the end face of the permanent magnet. The magnetic induction intensity in $z$ direction achieved maximum value on the end face of the permanent magnet, decreasing rapidly with the distance increased; the magnetic induction intensity in $x, y$ directions achieved maximum value at the edge of the permanent magnet end face, and zero at the center. So, considering the effects of seeds filling, the diameter of the cylindrical permanent magnet should be less than magnetic powder coated seed, meanwhile, the air gap between the permanent magnet end face and the magnetic powder coated seed should be smaller as much as possible.

Acknowledgements. This work was financially supported by Three Engineering of Agricultural Machinery, Jiangsu province (NJ2009-41), sponsored by Qing Lan Project of Jiangsu Province (Su teacher(2010)no.27) and A Project Funded by Priority Academic Program Development of Jiangsu Higher Education Institutions (Su financial teacher(2011)no.8).

\section{References}

1. Hu, J., Zheng, S., Liu, W.: Design and experiment of precision magnetic cylinder seeder. Transactions of the Chinese Society for Agricultural Machinery 40(3), 60-63 (2009)

2. Hu, J., Wang, X., Yan, J., Zheng, S.: Analysis for Magnetic Field Characteristics of Magnetic Seed-metering Elements and Structure Optimization. Transactions of the Chinese Society for Agricultural Machinery 42(2), 67-70 (2011)

3. Hu, J., Li, X., Mao, H.: Magnetic field characteristic analysis for the magnetic seed-metering space of the precision seeder. Transactions of the Chinese Society of Agricultural Engineering 21(12), 39-43 (2005)

4. Hu, J., Wang, Q., Shao, X.: Simulation on Magnetic Precision Seed-metering Device. Transactions of the Chinese Society for Agricultural Machinery 41(12), 35-38 (2010) 
5. Magrab, E.B., Azarm, S., Balachandran, B., et al.: An Engineer's Guide to MATLAB with Application from Mechanical, Aerospace, Electrical and Civil Engineering. Publishing House of Electronics Industry, Beijing (2006)

6. Zhang, X., Fang, L., Li, G.-L., Deng, F.-L., Li, J.-P.: Modeling and Simulation for Permanent Column in Wireless Endoscope. Journal of System Simulation 19(3), 494-497 (2007)

7. Hui, C.: Experimental Study on Magnetic Field Characteristic of Magnetic Marker. Wuhan Ligongdaxue, Wuhan (2007)

8. Kong, F., Chen, G., Cao, W.: Numerical calculation of magnetic field in magnetic couplings of magnetic pump. Chinese Journal of Mechanical Engineering 42(11), 213-217 (2006)

9. Liu, H.: Research of the Three-Dimensional Magnetic Field Distribution Around a Rectangular Permanent Magnet. Beijing Industrial University, Beijing (2006) 